\title{
UK Renal Registry 12th Annual Report (December 2009): Appendix E Additional Data Tables for 2008 new and existing patients
}

\section{E:1 Patients starting renal replacement therapy in 2008}

Table E.1.1. Take on totals for new patients on dialysis at 90 days in 2008

\begin{tabular}{lrrrrr}
\hline & \multicolumn{2}{c}{ Aged $<65$} & & \multicolumn{2}{c}{ Aged $\geqslant 65$} \\
\cline { 2 - 3 } \cline { 5 - 6 } \cline { 5 - 6 } & HD & PD & & HD & PD \\
& $\mathrm{N}$ & $\mathrm{N}$ & & $\mathrm{N}$ & $\mathrm{N}$ \\
\hline England & 1,803 & 737 & & 1,941 & 378 \\
N Ireland & 50 & 18 & & 73 & 7 \\
Scotland & 170 & 64 & & 213 & 34 \\
Wales & 107 & 45 & & 125 & 28 \\
UK & $\mathbf{2 , 1 3 0}$ & $\mathbf{8 6 4}$ & & $\mathbf{2 , 3 5 2}$ & $\mathbf{4 4 7}$ \\
\hline
\end{tabular}

Table E.1.2. Number of patients per treatment modality at 90 days

\begin{tabular}{lrrcrr}
\hline & HD & PD & Transplant & $\begin{array}{c}\text { Stopped } \\
\text { treatment }\end{array}$ & Died \\
\hline England & 3,744 & 1,115 & 357 & 15 & 333 \\
N Ireland & 123 & 25 & 2 & 6 & 10 \\
Scotland & 383 & 98 & 19 & 1 & 42 \\
Wales & 232 & 73 & 13 & 7 & 24 \\
UK & $\mathbf{4 , 4 8 2}$ & $\mathbf{1 , 3 1 1}$ & $\mathbf{3 9 1}$ & $\mathbf{2 9}$ & $\mathbf{4 0 9}$ \\
\hline
\end{tabular}

Table E.1.3. First treatment modality

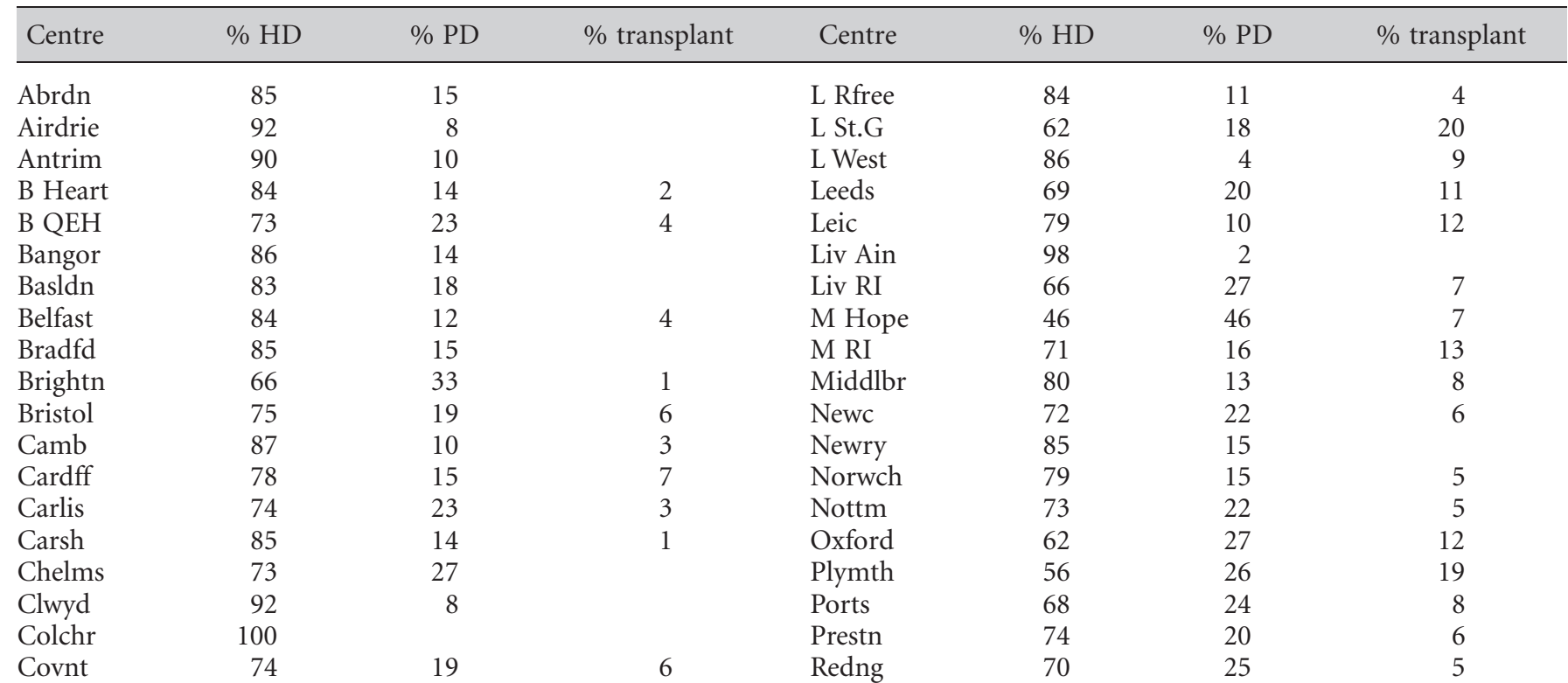

\section{KARGER}

Fax +41613061234

E-Mail karger@karger.ch

www.karger.com
C 2010 S. Karger AG, Basel

$1160-2110 / 10 / 1155-0338 \$ 26.00 / 0$

Accessible online at: www.karger.com/nec
UK Renal Registry, Southmead Hospital, Southmead Road, Bristol BS10 5NB, UK

Email: renalreg@renalreg.com 
Table E.1.3. Continued

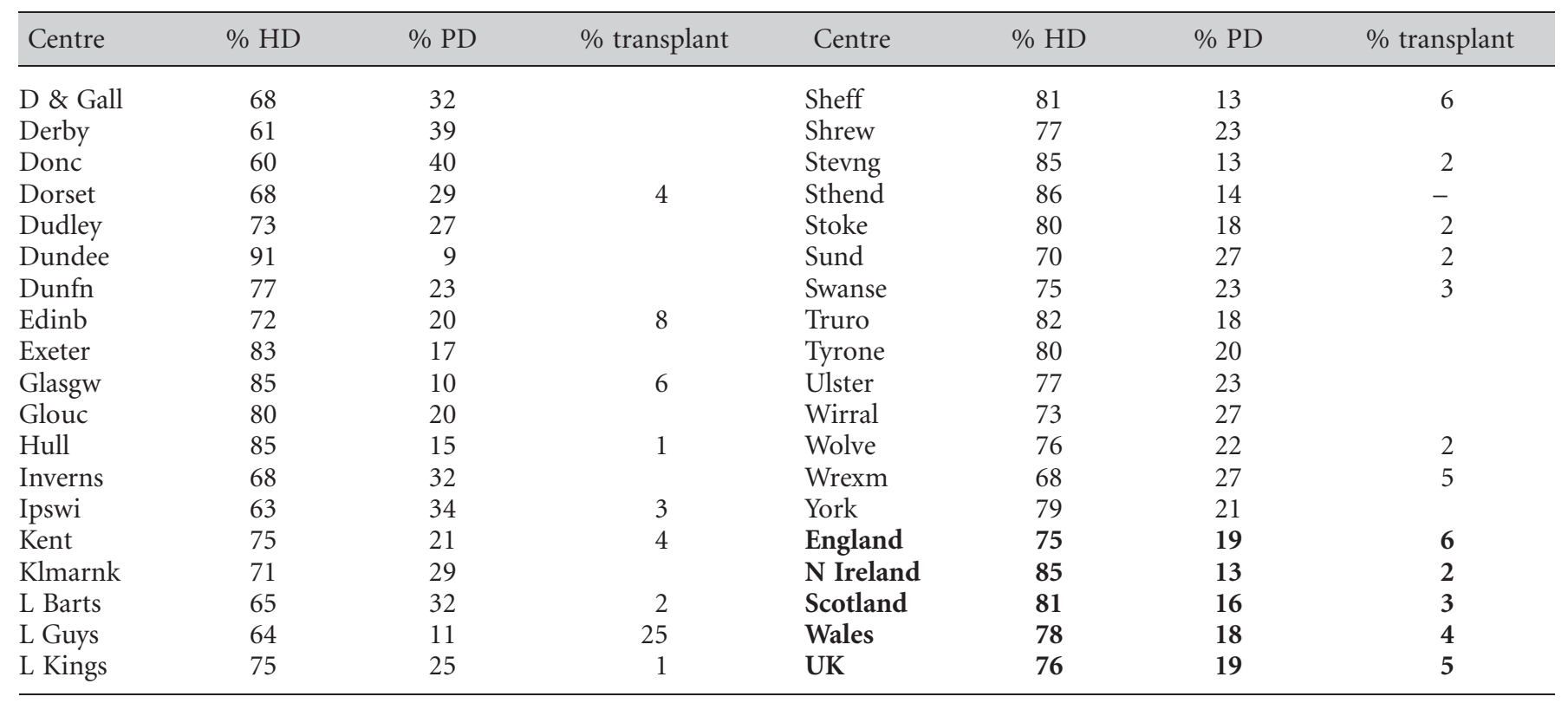

Table E.1.4. First treatment modality, patient numbers

\begin{tabular}{lrrr}
\hline & HD & PD & Transplant \\
\hline England & 4,180 & 1,085 & 320 \\
N Ireland & 147 & 23 & 3 \\
Scotland & 430 & 85 & 17 \\
Wales & 273 & 63 & 13 \\
UK & $\mathbf{5 , 0 3 0}$ & $\mathbf{1 , 2 5 6}$ & $\mathbf{3 5 3}$ \\
\hline
\end{tabular}

Table E.1.5. Gender breakdown by treatment modality (at 90 days)

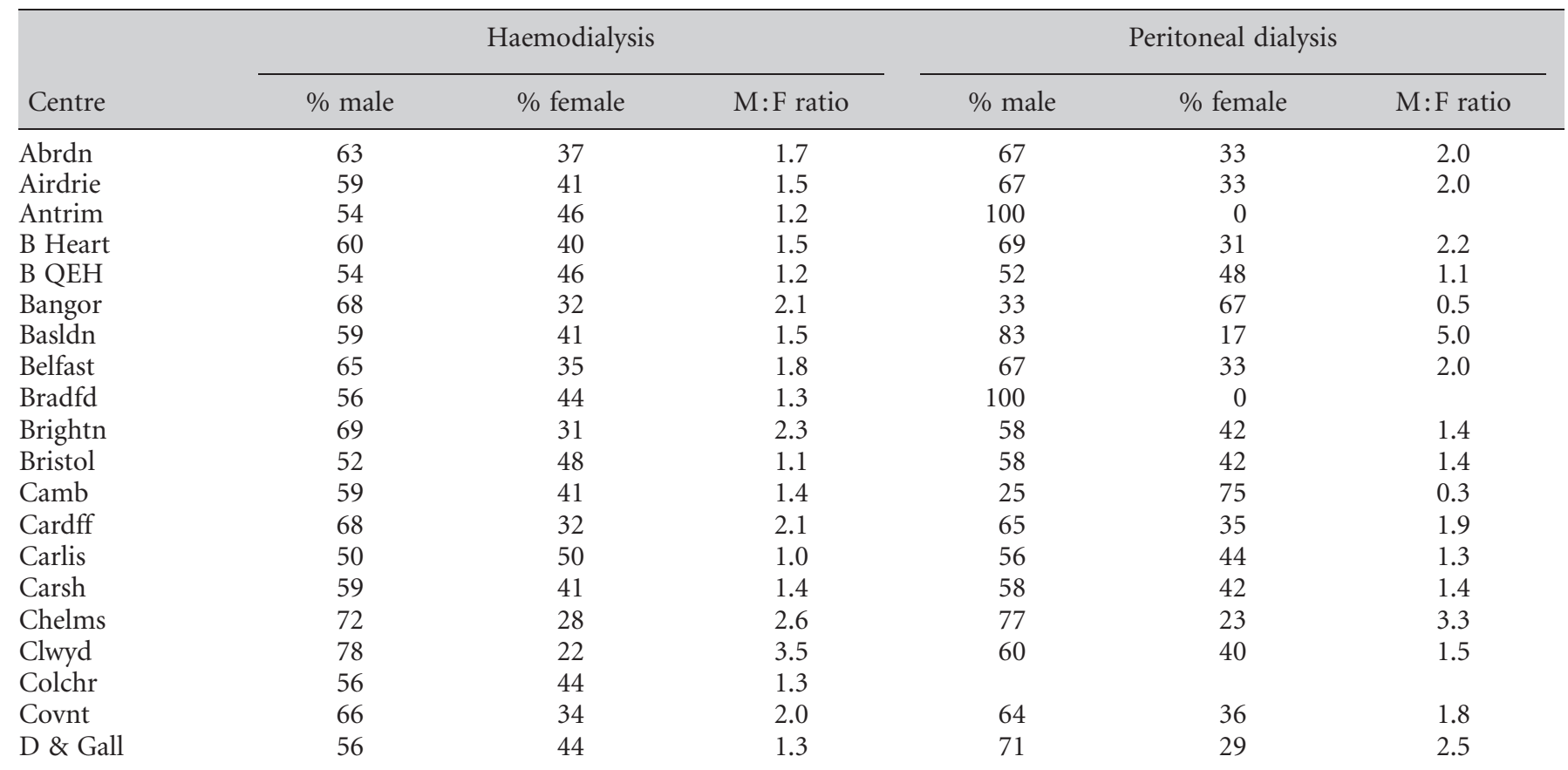


Table E.1.5. Gender breakdown by treatment modality (at 90 days)

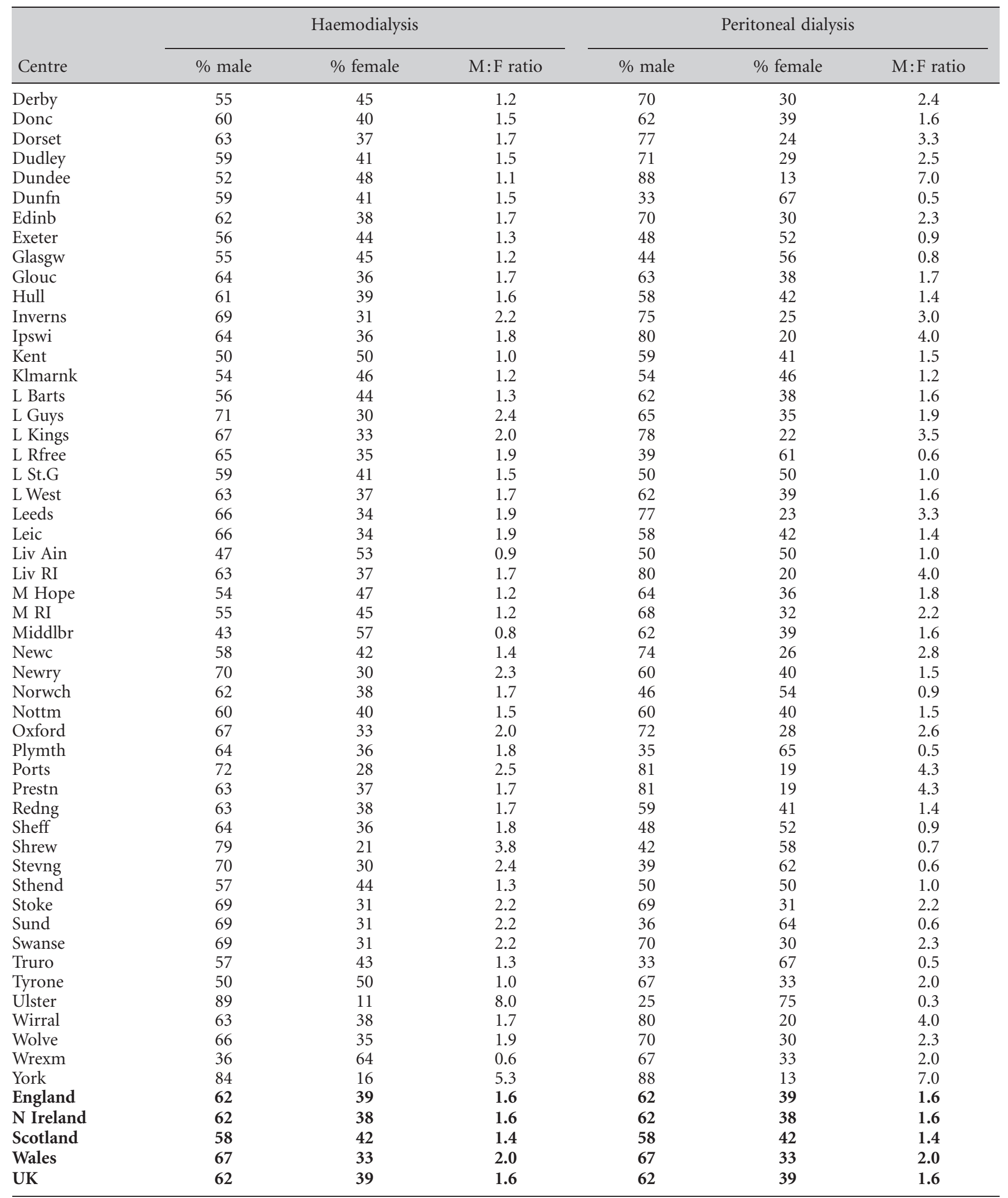




\section{E:2 Prevalent patients on 31/12/2008}

Table E.2.1. Treatment modalities for patients aged under and over 65

\begin{tabular}{|c|c|c|c|c|c|c|c|c|}
\hline \multirow[b]{2}{*}{ Centre } & \multicolumn{4}{|c|}{ Patients aged $<65$} & \multicolumn{4}{|c|}{ Patients aged $\geqslant 65$} \\
\hline & $\% \mathrm{HD}$ & $\% \mathrm{PD}$ & $\%$ transplant & $\mathrm{HD}: \mathrm{PD}$ & $\% \mathrm{HD}$ & $\% \mathrm{PD}$ & $\%$ transplant & $\mathrm{HD}: \mathrm{PD}$ \\
\hline Airdrie & 53 & 7 & 41 & 7.9 & 90 & 3 & 8 & 36.0 \\
\hline Antrim & 42 & 8 & 50 & 5.2 & 80 & 9 & 10 & 8.6 \\
\hline B Heart & 59 & 5 & 36 & 11.5 & 82 & 6 & 12 & 13.5 \\
\hline Basldn & 60 & 12 & 29 & 5.0 & 70 & 21 & 9 & 3.4 \\
\hline Belfast & 27 & 7 & 66 & 4.0 & 59 & 8 & 33 & 7.6 \\
\hline Bradfd & 39 & 8 & 53 & 4.9 & 66 & 8 & 26 & 8.2 \\
\hline Brightn & 30 & 12 & 58 & 2.6 & 64 & 15 & 21 & 4.3 \\
\hline Bristol & 24 & 7 & 69 & 3.4 & 62 & 7 & 31 & 8.5 \\
\hline Camb & 20 & 6 & 74 & 3.7 & 70 & 4 & 26 & 17.0 \\
\hline Clwyd & 42 & 7 & 51 & 6.0 & 71 & 7 & 22 & 10.7 \\
\hline Colchr & 100 & & & 0.0 & 100 & & & 0.0 \\
\hline Covnt & 33 & 8 & 59 & 4.0 & 61 & 15 & 24 & 4.1 \\
\hline D \& Gall & 24 & 15 & 61 & 1.6 & 79 & 13 & 9 & 6.2 \\
\hline Derby & 55 & 21 & 24 & 2.6 & 70 & 19 & 10 & 3.6 \\
\hline Derry & 44 & 5 & 51 & 8.7 & 76 & 8 & 16 & 9.3 \\
\hline Donc & 42 & 24 & 34 & 1.8 & 67 & 28 & 5 & 2.4 \\
\hline Dorset & 31 & 7 & 62 & 4.6 & 55 & 16 & 29 & 3.4 \\
\hline Dudley & 46 & 22 & 32 & 2.1 & 60 & 17 & 22 & 3.4 \\
\hline Dundee & 30 & 8 & 62 & 3.9 & 66 & 6 & 28 & 11.4 \\
\hline Dunfn & 40 & 10 & 50 & 4.1 & 71 & 15 & 15 & 4.8 \\
\hline Klmarnk & 41 & 15 & 44 & 2.7 & 77 & 17 & 5 & 4.5 \\
\hline L Barts & 36 & 13 & 50 & 2.7 & 57 & 20 & 23 & 2.8 \\
\hline L Guys & 27 & 3 & 70 & 8.4 & 62 & 5 & 32 & 11.5 \\
\hline L Kings & 44 & 9 & 47 & 4.7 & 71 & 13 & 16 & 5.6 \\
\hline L Rfree & 32 & 6 & 62 & 5.8 & 67 & 7 & 25 & 9.3 \\
\hline L St.G & 24 & 6 & 70 & 4.0 & 59 & 14 & 27 & 4.1 \\
\hline L West & 35 & 1 & 63 & 25.3 & 75 & 2 & 23 & 31.4 \\
\hline Leeds & 25 & 8 & 67 & 3.1 & 65 & 7 & 28 & 9.3 \\
\hline Leic & 34 & 8 & 58 & 4.3 & 65 & 14 & 22 & 4.8 \\
\hline Liv Ain & 96 & 4 & & 22.0 & 100 & & & 0.0 \\
\hline Liv RI & 27 & 8 & 65 & 3.5 & 51 & 12 & 37 & 4.3 \\
\hline M Hope & 33 & 17 & 50 & 1.9 & 63 & 20 & 18 & 3.2 \\
\hline M RI & 24 & 6 & 71 & 4.2 & 50 & 12 & 38 & 4.0 \\
\hline Middlbr & 30 & 4 & 67 & 8.2 & 68 & 3 & 29 & 20.0 \\
\hline Newc & 23 & 6 & 71 & 4.1 & 49 & 6 & 44 & 8.1 \\
\hline Newry & 49 & 11 & 39 & 4.3 & 77 & 3 & 20 & 27.5 \\
\hline Norwch & 36 & 12 & 52 & 3.1 & 77 & 11 & 12 & 7.2 \\
\hline
\end{tabular}


Table E.2.1. Continued

\begin{tabular}{|c|c|c|c|c|c|c|c|c|}
\hline \multirow[b]{2}{*}{ Centre } & \multicolumn{4}{|c|}{ Patients aged $<65$} & \multicolumn{4}{|c|}{ Patients aged $\geqslant 65$} \\
\hline & $\% \mathrm{HD}$ & $\% \mathrm{PD}$ & $\%$ transplant & HD:PD & $\% \mathrm{HD}$ & $\% \mathrm{PD}$ & $\%$ transplant & HD:PD \\
\hline Nottm & 29 & 13 & 58 & 2.2 & 71 & 13 & 15 & 5.3 \\
\hline Oxford & 19 & 7 & 74 & 2.7 & 48 & 15 & 37 & 3.2 \\
\hline Plymth & 17 & 10 & 74 & 1.8 & 50 & 16 & 34 & 3.2 \\
\hline Ports & 24 & 6 & 70 & 3.7 & 62 & 10 & 28 & 6.5 \\
\hline Prestn & 40 & 8 & 53 & 5.2 & 74 & 6 & 20 & 11.7 \\
\hline Redng & 31 & 14 & 55 & 2.2 & 66 & 13 & 20 & 4.9 \\
\hline Sheff & 37 & 5 & 58 & 7.2 & 73 & 9 & 19 & 8.4 \\
\hline Shrew & 43 & 13 & 44 & 3.4 & 77 & 9 & 13 & 8.3 \\
\hline Stevng & 51 & 7 & 42 & 7.5 & 80 & 7 & 13 & 11.2 \\
\hline Sthend & 49 & 11 & 40 & 4.6 & 83 & 4 & 13 & 19.0 \\
\hline Stoke & 36 & 12 & 52 & 3.0 & 62 & 15 & 24 & 4.2 \\
\hline Sund & 37 & 6 & 57 & 6.5 & 71 & 9 & 20 & 7.9 \\
\hline Swanse & 46 & 10 & 44 & 4.5 & 75 & 14 & 11 & 5.5 \\
\hline Truro & 28 & 11 & 61 & 2.4 & 70 & 8 & 22 & 8.4 \\
\hline Tyrone & 49 & 10 & 41 & 5.0 & 89 & 2 & 9 & 49.0 \\
\hline Ulster & 78 & 8 & 14 & 9.3 & 95 & 3 & 2 & 28.0 \\
\hline Wirral & 82 & 18 & & 4.4 & 84 & 16 & & 5.4 \\
\hline Wolve & 48 & 13 & 39 & 3.8 & 80 & 13 & 7 & 6.3 \\
\hline Wrexm & 27 & 5 & 67 & 5.0 & 47 & 22 & 30 & 2.1 \\
\hline York & 32 & 4 & 64 & 7.9 & 65 & 14 & 21 & 4.7 \\
\hline England & 32 & 8 & 59 & 4.0 & 67 & 11 & 22 & 6.2 \\
\hline N Ireland & 36 & 8 & 56 & 4.8 & 74 & 6 & 20 & 11.6 \\
\hline Scotland & 33 & 7 & 59 & 4.5 & 69 & 9 & 22 & 7.7 \\
\hline Wales & 30 & 8 & 61 & 3.6 & 66 & 14 & 19 & 4.7 \\
\hline UK & 32 & 8 & 59 & 4.0 & 67 & 11 & 22 & 6.3 \\
\hline
\end{tabular}

Table E.2.2. Number of patients under and over 65 per treatment modality

\begin{tabular}{lrrrrrrrr}
\hline & \multicolumn{3}{c}{ Patients aged $<65$} & & \multicolumn{3}{c}{ Patients aged $\geqslant 65$} \\
\cline { 2 - 5 } \cline { 7 - 8 } & \multicolumn{1}{c}{ HD } & PD & Transplant & & HD & PD & Transplant \\
\hline England & 8,535 & 2,152 & 15,648 & & 8,769 & 1,412 & 2,915 \\
N Ireland & 324 & 68 & 506 & & 395 & 34 & 104 \\
Scotland & 954 & 214 & 1,696 & & 881 & 114 & 283 \\
Wales & 483 & 134 & 976 & & 586 & 125 & 172 \\
UK & $\mathbf{1 0 , 2 9 6}$ & $\mathbf{2 , 5 6 8}$ & $\mathbf{1 8 , 8 2 6}$ & & $\mathbf{1 0 , 6 3 1}$ & $\mathbf{1 , 6 8 5}$ & $\mathbf{3 , 4 7 4}$ \\
\hline
\end{tabular}


Table E.2.3. Dialysis modalities for patients aged under 65

\begin{tabular}{|c|c|c|c|c|c|c|c|c|}
\hline Centre & $\begin{array}{c}\% \\
\text { home } \\
\text { HD }\end{array}$ & $\begin{array}{c}\% \\
\text { hospital } \\
\text { HD }\end{array}$ & $\begin{array}{c}\% \\
\text { satellite } \\
\text { HD }\end{array}$ & $\begin{array}{c}\% \\
\text { connect } \\
\text { PD }\end{array}$ & $\begin{array}{c}\% \\
\text { disconnect } \\
\text { PD }\end{array}$ & $\begin{array}{c}\% \\
\text { cycling PD } \\
\geqslant 6 \text { nights }\end{array}$ & $\begin{array}{c}\% \\
\text { cycling PD } \\
<6 \text { nights }\end{array}$ & $\begin{array}{c}\% \\
\text { unknown } \\
\text { type of PD }\end{array}$ \\
\hline Airdrie $^{*}$ & 0 & 89 & 0 & 0 & 1 & 10 & 0 & 0 \\
\hline Antrim & 4 & 80 & 0 & 0 & 2 & 14 & 0 & 0 \\
\hline B Heart & 4 & 81 & 7 & 0 & 8 & 0 & 0 & 0 \\
\hline Basldn & 0 & 83 & 0 & 0 & 4 & 12 & 0 & 0 \\
\hline Belfast & 2 & 77 & 1 & 0 & 3 & 15 & 0 & 1 \\
\hline Bradfd & 0 & 59 & 24 & 0 & 7 & 10 & 0 & 0 \\
\hline Brightn & 11 & 38 & 23 & 0 & 11 & 17 & 0 & 0 \\
\hline Bristol & 8 & 14 & 55 & 0 & 16 & 7 & 0 & 0 \\
\hline Chelms & 0 & 70 & 0 & 0 & 17 & 11 & 2 & 0 \\
\hline Clwyd & 2 & 84 & 0 & 0 & 8 & 6 & 0 & 0 \\
\hline Colchr & 0 & 100 & 0 & 0 & 0 & 0 & 0 & 0 \\
\hline Covnt & 3 & 77 & 0 & 0 & 20 & 0 & 0 & 0 \\
\hline D \& Gall ${ }^{*}$ & 0 & 62 & 0 & 0 & 4 & 19 & 15 & 0 \\
\hline Derby & 5 & 67 & 0 & 0 & 25 & 3 & 0 & 0 \\
\hline Derry & 0 & 86 & 3 & 3 & 0 & 7 & 0 & 0 \\
\hline Donc & 0 & 64 & 0 & 0 & 16 & 18 & 2 & 0 \\
\hline Dorset & 0 & 25 & 57 & 0 & 5 & 12 & 1 & 0 \\
\hline Dudley & 2 & 48 & 19 & 0 & 32 & 0 & 0 & 0 \\
\hline Dundee $^{*}$ & 0 & 80 & 0 & 0 & 2 & 17 & 1 & 0 \\
\hline Dunfn $^{\star}$ & 0 & 81 & 0 & 0 & 4 & 15 & 0 & 0 \\
\hline Klmarnk $^{*}$ & 1 & 72 & 0 & 0 & 5 & 13 & 9 & 0 \\
\hline L Barts & 1 & 40 & 32 & 0 & 7 & 20 & 0 & 0 \\
\hline L Guys & 9 & 25 & 55 & 0 & 5 & 0 & 6 & 0 \\
\hline L Kings & 0 & 29 & 54 & 0 & 4 & 13 & 0 & 0 \\
\hline L Rfree & 3 & 38 & 45 & 0 & 4 & 10 & 0 & 0 \\
\hline L St.G & 4 & 62 & 14 & 2 & 4 & 14 & 0 & 0 \\
\hline L West & 1 & 30 & 65 & 0 & 2 & 2 & 0 & 0 \\
\hline Leeds & 5 & 14 & 57 & 0 & 8 & 16 & 0 & 0 \\
\hline Leic & 4 & 22 & 56 & 0 & 8 & 11 & 0 & 0 \\
\hline Liv Ain & 6 & 7 & 83 & 0 & 0 & 4 & 0 & 0 \\
\hline Liv RI & 2 & 39 & 37 & 0 & 7 & 15 & 0 & 0 \\
\hline M Hope & 3 & 34 & 29 & 0 & 24 & 9 & 0 & 1 \\
\hline M RI & 16 & 26 & 38 & 0 & 4 & 13 & 2 & 0 \\
\hline Middlbr & 2 & 34 & 53 & 0 & 10 & 1 & 0 & 0 \\
\hline Newc & 5 & 75 & 0 & 0 & 3 & 17 & 0 & 0 \\
\hline Newry & 2 & 79 & 0 & 0 & 0 & 19 & 0 & 0 \\
\hline Norwch & 5 & 45 & 26 & 0 & 23 & 0 & 1 & 0 \\
\hline Nottm & 2 & 45 & 22 & 0 & 10 & 21 & 0 & 0 \\
\hline Oxford & 5 & 67 & 0 & 0 & 10 & 18 & 0 & 0 \\
\hline
\end{tabular}


Table E.2.3. Dialysis modalities for patients aged under 65

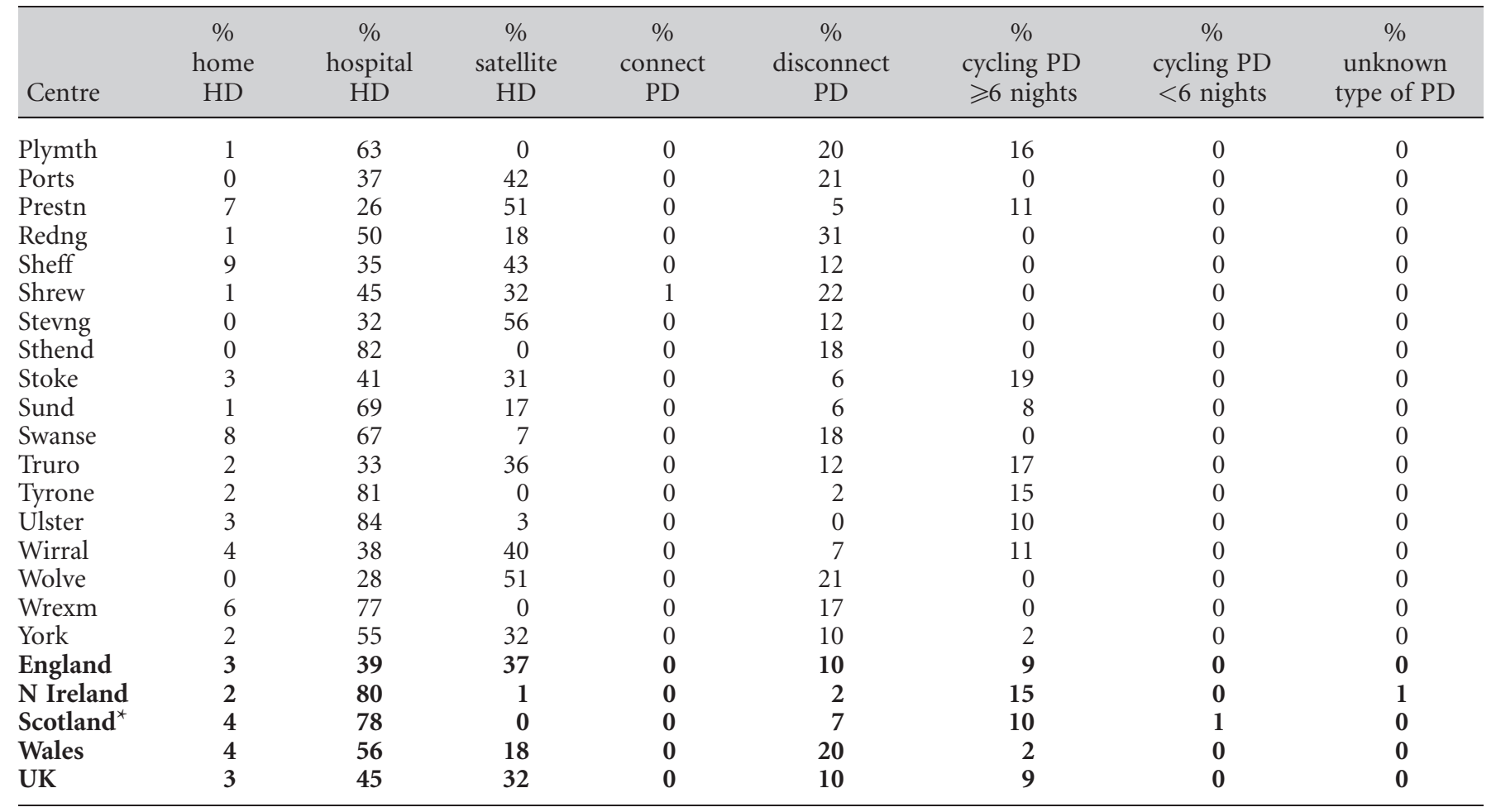

Note: ${ }^{\star}$ All haemodialysis patients in centres in Scotland are shown as receiving treatment in home or hospital as no information is available regarding numbers using satellite dialysis centres

Table E.2.4. Dialysis modalities for patients aged over 65

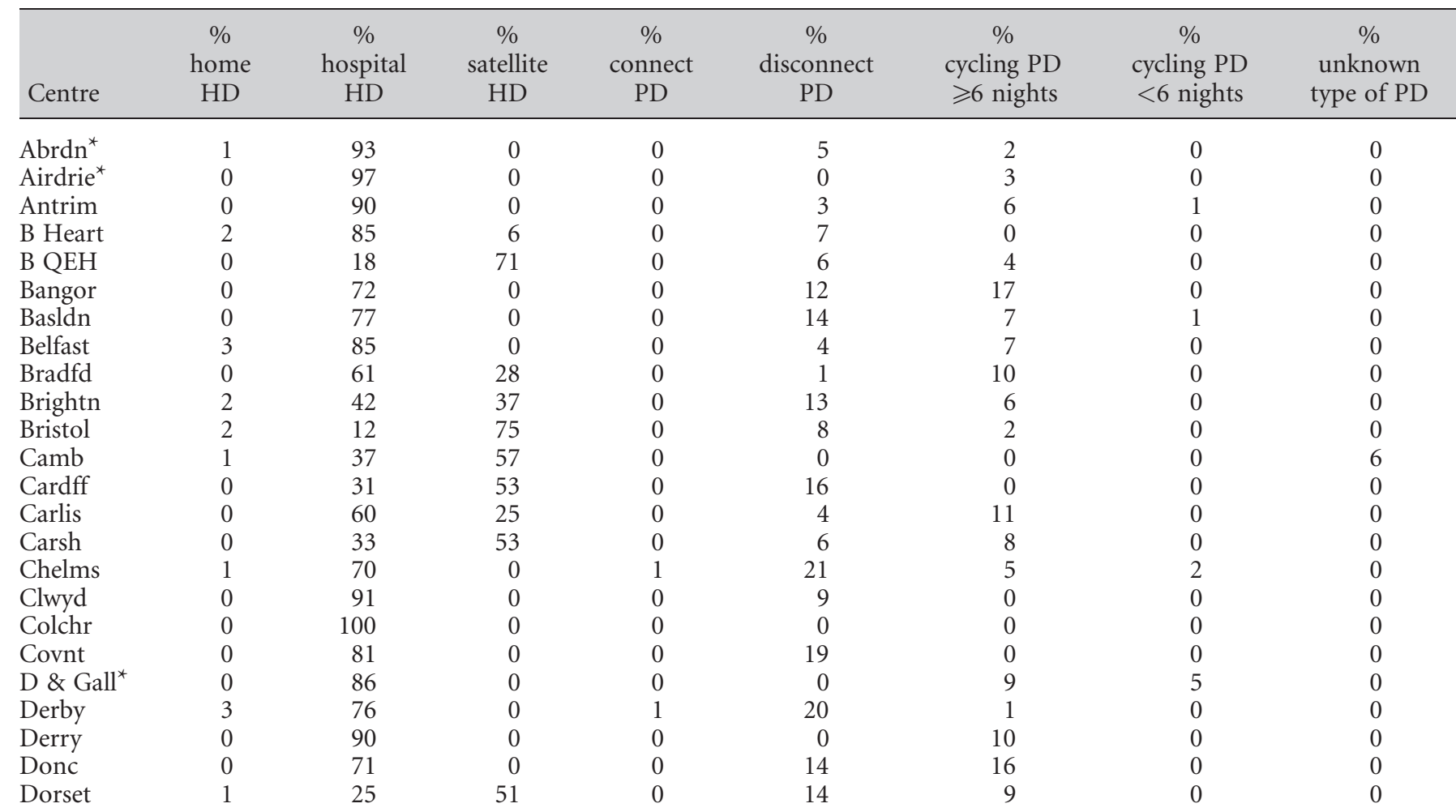


Table E.2.4. Continued

\begin{tabular}{|c|c|c|c|c|c|c|c|c|}
\hline Centre & $\begin{array}{c}\% \\
\text { home } \\
\text { HD }\end{array}$ & $\begin{array}{c}\% \\
\text { hospital } \\
\text { HD }\end{array}$ & $\begin{array}{c}\% \\
\text { satellite } \\
\text { HD }\end{array}$ & $\begin{array}{c}\% \\
\text { connect } \\
\text { PD }\end{array}$ & $\begin{array}{c}\% \\
\text { disconnect } \\
\text { PD }\end{array}$ & $\begin{array}{c}\% \\
\text { cycling PD } \\
\geqslant 6 \text { nights }\end{array}$ & $\begin{array}{c}\% \\
\text { cycling PD } \\
<6 \text { nights }\end{array}$ & $\begin{array}{c}\% \\
\text { unknown } \\
\text { type of PD }\end{array}$ \\
\hline Dudley & 0 & 59 & 19 & 0 & 23 & 0 & 0 & 0 \\
\hline Dundee ${ }^{*}$ & 0 & 92 & 0 & 0 & 0 & 7 & 1 & 0 \\
\hline $\operatorname{Dunfn}^{*}$ & 0 & 83 & 0 & 0 & 0 & 17 & 0 & 0 \\
\hline Edinb $^{*}$ & 0 & 82 & 0 & 0 & 8 & 10 & 0 & 0 \\
\hline Exeter & 0 & 38 & 45 & 0 & 14 & 3 & 0 & 0 \\
\hline Glasgw $^{*}$ & 1 & 91 & 0 & 0 & 5 & 2 & 0 & 0 \\
\hline Glouc & 0 & 90 & 0 & 0 & 3 & 8 & 0 & 0 \\
\hline Hull & 2 & 42 & 42 & 0 & 5 & 8 & 0 & 0 \\
\hline Inverns ${ }^{*}$ & 2 & 76 & 0 & 0 & 10 & 13 & 0 & 0 \\
\hline Ipswi & 0 & 66 & 0 & 0 & 22 & 11 & 0 & 2 \\
\hline Kent & 0 & 25 & 59 & 0 & 15 & 0 & 0 & 0 \\
\hline Klmarnk $^{*}$ & 0 & 82 & 0 & 0 & 8 & 5 & 6 & 0 \\
\hline L Barts & 0 & 37 & 37 & 0 & 12 & 14 & 0 & 0 \\
\hline L Guys & 0 & 26 & 66 & 0 & 4 & 0 & 4 & 0 \\
\hline L Kings & 0 & 23 & 62 & 0 & 5 & 10 & 0 & 0 \\
\hline L Rfree & 1 & 36 & 53 & 0 & 4 & 6 & 0 & 0 \\
\hline L St.G & 0 & 60 & 20 & 3 & 6 & 11 & 0 & 0 \\
\hline L West & 0 & 27 & 70 & 0 & 2 & 1 & 0 & 0 \\
\hline Leeds & 0 & 10 & 80 & 0 & 4 & 6 & 0 & 0 \\
\hline Leic & 0 & 26 & 56 & 0 & 9 & 9 & 0 & 0 \\
\hline Liv Ain & 0 & 18 & 82 & 0 & 0 & 0 & 0 & 0 \\
\hline Liv RI & 0 & 48 & 33 & 0 & 11 & 5 & 2 & 0 \\
\hline M Hope & 0 & 43 & 33 & 0 & 20 & 3 & 0 & 1 \\
\hline M RI & 3 & 29 & 49 & 1 & 6 & 9 & 5 & 0 \\
\hline Middlbr & 1 & 30 & 64 & 0 & 5 & 0 & 0 & 0 \\
\hline Newc & 0 & 89 & 0 & 0 & 1 & 10 & 0 & 0 \\
\hline Newry & 2 & 95 & 0 & 0 & 0 & 4 & 0 & 0 \\
\hline Norwch & 1 & 51 & 36 & 0 & 10 & 1 & 1 & 0 \\
\hline Nottm & 1 & 48 & 35 & 0 & 8 & 8 & 0 & 0 \\
\hline Oxford & 2 & 74 & 1 & 0 & 17 & 7 & 0 & 0 \\
\hline Plymth & 0 & 76 & 0 & 0 & 18 & 6 & 0 & 0 \\
\hline Ports & 0 & 28 & 59 & 0 & 13 & 0 & 0 & 0 \\
\hline Prestn & 1 & 20 & 71 & 0 & 4 & 4 & 0 & 0 \\
\hline Redng & 0 & 64 & 19 & 0 & 17 & 0 & 0 & 0 \\
\hline Sheff & 3 & 43 & 43 & 0 & 11 & 0 & 0 & 0 \\
\hline Shrew & 0 & 59 & 30 & 0 & 11 & 0 & 0 & 0 \\
\hline Stevng & 0 & 30 & 62 & 0 & 8 & 0 & 0 & 0 \\
\hline Sthend & 0 & 95 & 0 & 0 & 5 & 0 & 0 & 0 \\
\hline Stoke & 0 & 55 & 26 & 0 & 8 & 11 & 0 & 0 \\
\hline Sund & 0 & 66 & 23 & 0 & 10 & 1 & 0 & 0 \\
\hline Swanse & 0 & 67 & 17 & 0 & 15 & 0 & 0 & 0 \\
\hline Truro & 2 & 42 & 45 & 0 & 5 & 5 & 0 & 0 \\
\hline Tyrone & 0 & 98 & 0 & 0 & 0 & 2 & 0 & 0 \\
\hline Ulster & 0 & 97 & 0 & 0 & 0 & 3 & 0 & 0 \\
\hline Wirral & 0 & 38 & 46 & 0 & 6 & 10 & 0 & 0 \\
\hline Wolve & 0 & 23 & 64 & 0 & 13 & 1 & 0 & 0 \\
\hline Wrexm & 2 & 66 & 0 & 0 & 30 & 0 & 2 & 0 \\
\hline York & 0 & 49 & 34 & 0 & 18 & 0 & 0 & 0 \\
\hline England & 1 & 41 & 44 & 0 & 9 & 4 & 0 & 0 \\
\hline N Ireland & 1 & 91 & 0 & 0 & 2 & 6 & 0 & 0 \\
\hline Scotland ${ }^{*}$ & 1 & 88 & 0 & 0 & 5 & 6 & 1 & 0 \\
\hline Wales & 0 & 52 & 30 & 0 & 16 & 1 & 0 & 0 \\
\hline UK & 1 & 48 & 38 & 0 & 9 & 4 & 0 & 0 \\
\hline
\end{tabular}

Note: ${ }^{*}$ All haemodialysis patients in centres in Scotland are shown as receiving treatment in home or hospital as no information is available regarding numbers using satellite dialysis centres 
Table E.2.5. Patient age ranges by centre (\%)

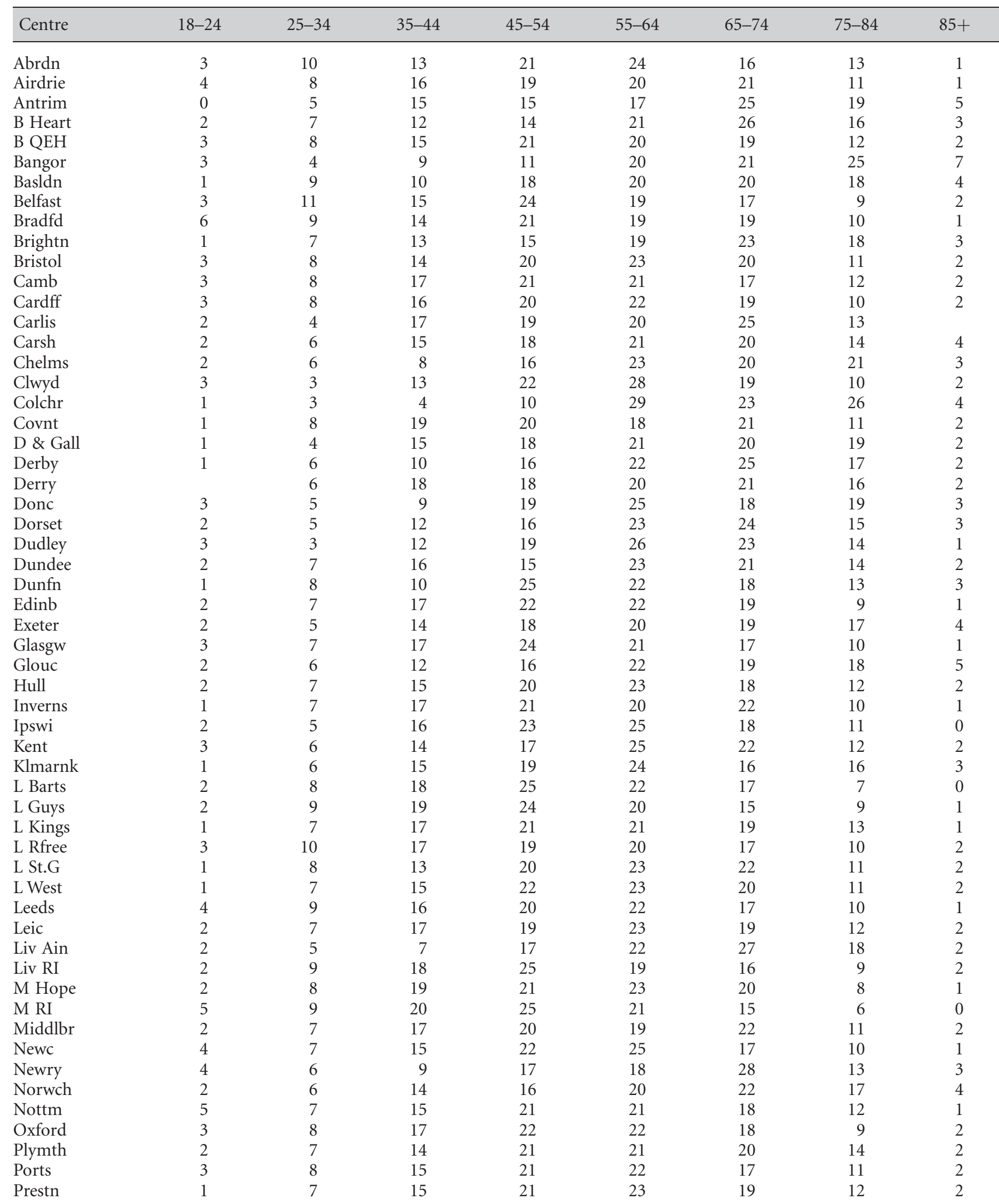


Table E.2.5. Continued

\begin{tabular}{lcccccccc}
\hline Centre & $18-24$ & $25-34$ & $35-44$ & $45-54$ & $55-64$ & $65-74$ & $75-84$ & $85+$ \\
\hline Redng & 2 & 5 & 14 & 18 & 22 & 22 & 15 & 3 \\
Sheff & 2 & 6 & 14 & 20 & 21 & 22 & 12 & 2 \\
Shrew & 4 & 6 & 13 & 17 & 21 & 22 & 14 & 2 \\
Stevng & 2 & 7 & 14 & 17 & 19 & 25 & 15 & 2 \\
Sthend & 1 & 6 & 8 & 15 & 25 & 23 & 16 & 6 \\
Stoke & 4 & 7 & 17 & 20 & 17 & 21 & 12 & 2 \\
Sund & 3 & 6 & 17 & 22 & 22 & 18 & 10 & 1 \\
Swanse & 2 & 5 & 10 & 14 & 24 & 24 & 18 & 3 \\
Truro & 1 & 4 & 12 & 13 & 21 & 23 & 22 & 4 \\
Tyrone & 3 & 7 & 15 & 9 & 25 & 22 & 15 & 3 \\
Ulster & & 3 & 6 & 11 & 18 & 35 & 22 & 5 \\
Wirral & 3 & 5 & 8 & 17 & 19 & 25 & 19 & 3 \\
Wolve & 2 & 6 & 14 & 19 & 17 & 23 & 17 & 2 \\
Wrexm & 5 & 6 & 17 & 21 & 17 & 20 & 13 & 1 \\
York & 4 & 8 & 14 & 15 & 21 & 18 & 16 & 4 \\
England & $\mathbf{2}$ & $\mathbf{7}$ & $\mathbf{1 5}$ & $\mathbf{2 0}$ & $\mathbf{2 1}$ & $\mathbf{1 9}$ & $\mathbf{1 2}$ & $\mathbf{2}$ \\
N Ireland & $\mathbf{2}$ & $\mathbf{8}$ & $\mathbf{1 4}$ & $\mathbf{1 9}$ & $\mathbf{1 9}$ & $\mathbf{2 2}$ & $\mathbf{1 3}$ & $\mathbf{3}$ \\
Scotland & $\mathbf{2}$ & $\mathbf{7}$ & $\mathbf{1 6}$ & $\mathbf{2 1}$ & $\mathbf{2 2}$ & $\mathbf{1 8}$ & $\mathbf{1 1}$ & $\mathbf{1}$ \\
Wales & $\mathbf{3}$ & $\mathbf{6}$ & $\mathbf{1 4}$ & $\mathbf{1 8}$ & $\mathbf{2 2}$ & $\mathbf{2 0}$ & $\mathbf{1 3}$ & $\mathbf{2}$ \\
UK & $\mathbf{2}$ & $\mathbf{7}$ & $\mathbf{1 5}$ & $\mathbf{2 0}$ & $\mathbf{2 1}$ & $\mathbf{1 9}$ & $\mathbf{1 2}$ & $\mathbf{2}$ \\
\hline
\end{tabular}

Table E.2.6. Dialysis modalities for non-diabetic patients (all ages)

\begin{tabular}{|c|c|c|c|c|c|c|c|c|}
\hline Centre & $\begin{array}{c}\% \\
\text { home } \\
\text { HD }\end{array}$ & $\begin{array}{c}\% \\
\text { hospital } \\
\text { HD }\end{array}$ & $\begin{array}{c}\% \\
\text { satellite } \\
\text { HD }\end{array}$ & $\begin{array}{c}\% \\
\text { connect } \\
\text { PD }\end{array}$ & $\begin{array}{c}\% \\
\text { disconnect } \\
\text { PD }\end{array}$ & $\begin{array}{c}\% \\
\text { cycling PD } \\
\geqslant 6 \text { nights }\end{array}$ & $\begin{array}{c}\% \\
\text { cycling PD } \\
<6 \text { nights }\end{array}$ & $\begin{array}{c}\% \\
\text { unknown } \\
\text { type of PD }\end{array}$ \\
\hline $\operatorname{Abrdn}^{*}$ & 3 & 81 & 0 & 0 & 9 & 6 & 0 & 0 \\
\hline Airdrie $^{*}$ & 0 & 92 & 0 & 0 & 1 & 7 & 0 & 0 \\
\hline Antrim & 2 & 85 & 0 & 0 & 2 & 10 & 1 & 0 \\
\hline B Heart & 4 & 80 & 7 & 0 & 9 & 0 & 0 & 0 \\
\hline B QEH & 2 & 20 & 61 & 0 & 9 & 7 & 0 & 0 \\
\hline Bangor & 4 & 66 & 0 & 0 & 13 & 18 & 0 & 0 \\
\hline Basldn & 0 & 82 & 0 & 0 & 9 & 9 & 1 & 0 \\
\hline Belfast & 2 & 80 & 0 & 0 & 4 & 13 & 0 & 1 \\
\hline Bradfd & 0 & 53 & 31 & 0 & 4 & 11 & 0 & 0 \\
\hline Brightn & 6 & 40 & 33 & 0 & 12 & 10 & 0 & 0 \\
\hline Bristol & 6 & 11 & 66 & 0 & 13 & 5 & 0 & 0 \\
\hline Camb & 1 & 36 & 50 & 0 & 0 & 0 & 0 & 13 \\
\hline Cardff & 0 & 35 & 42 & 0 & 22 & 0 & 0 & 0 \\
\hline Carlis & 0 & 50 & 25 & 0 & 6 & 19 & 0 & 0 \\
\hline Carsh & 0 & 33 & 49 & 0 & 7 & 11 & 0 & 0 \\
\hline Chelms & 1 & 68 & 0 & 0 & 20 & 10 & 1 & 0 \\
\hline Clwyd & 2 & 87 & 0 & 0 & 8 & 3 & 0 & 0 \\
\hline Colchr & 0 & 100 & 0 & 0 & 0 & 0 & 0 & 0 \\
\hline Covnt & 2 & 77 & 0 & 0 & 21 & 0 & 0 & 0 \\
\hline D \& Gall ${ }^{*}$ & 0 & 75 & 0 & 0 & 0 & 14 & 11 & 0 \\
\hline Derby & 5 & 72 & 0 & 0 & 22 & 2 & 0 & 0 \\
\hline Derry & 0 & 90 & 0 & 2 & 0 & 8 & 0 & 0 \\
\hline Donc & 0 & 70 & 0 & 0 & 14 & 16 & 1 & 0 \\
\hline Dorset & 0 & 24 & 54 & 0 & 11 & 11 & 0 & 0 \\
\hline Dudley & 1 & 54 & 20 & 0 & 25 & 0 & 0 & 0 \\
\hline Dundee & 0 & 83 & 0 & 0 & 1 & 14 & 1 & 0 \\
\hline $\operatorname{Dunfn}^{*}$ & 0 & 78 & 0 & 0 & 3 & 19 & 0 & 0 \\
\hline Edinb $^{*}$ & 3 & 75 & 0 & 0 & 10 & 13 & 0 & 0 \\
\hline
\end{tabular}


Table E.2.6. Continued

\begin{tabular}{|c|c|c|c|c|c|c|c|c|}
\hline Centre & $\begin{array}{c}\% \\
\text { home } \\
\text { HD }\end{array}$ & $\begin{array}{c}\% \\
\text { hospital } \\
\text { HD }\end{array}$ & $\begin{array}{c}\% \\
\text { satellite } \\
\text { HD }\end{array}$ & $\begin{array}{c}\% \\
\text { connect } \\
\text { PD }\end{array}$ & $\begin{array}{c}\% \\
\text { disconnect } \\
\text { PD }\end{array}$ & $\begin{array}{c}\% \\
\text { cycling PD } \\
\geqslant 6 \text { nights }\end{array}$ & $\begin{array}{c}\% \\
\text { cycling PD } \\
<6 \text { nights }\end{array}$ & $\begin{array}{c}\% \\
\text { unknown } \\
\text { type of PD }\end{array}$ \\
\hline Exeter & 0 & 31 & 46 & 0 & 15 & 7 & 0 & 0 \\
\hline Glasgw $^{*}$ & 5 & 86 & 0 & 0 & 6 & 3 & 0 & 0 \\
\hline Glouc & 0 & 84 & 0 & 0 & 4 & 12 & 0 & 0 \\
\hline Hull & 4 & 39 & 37 & 0 & 8 & 13 & 0 & 0 \\
\hline Inverns ${ }^{\star}$ & 4 & 66 & 0 & 0 & 9 & 20 & 0 & 0 \\
\hline Ipswi & 2 & 63 & 0 & 0 & 21 & 13 & 0 & 1 \\
\hline Kent & 1 & 22 & 57 & 0 & 20 & 0 & 0 & 0 \\
\hline Klmarnk ${ }^{*}$ & 1 & 76 & 0 & 0 & 8 & 10 & 6 & 0 \\
\hline L Barts & 1 & 37 & 34 & 0 & 9 & 19 & 0 & 0 \\
\hline L Guys & 6 & 23 & 61 & 0 & 4 & 0 & 6 & 0 \\
\hline L Kings & 0 & 25 & 56 & 0 & 6 & 13 & 0 & 0 \\
\hline L Rfree & 3 & 36 & 49 & 0 & 4 & 7 & 0 & 0 \\
\hline L St.G & 3 & 61 & 15 & 3 & 6 & 12 & 0 & 0 \\
\hline L West & 1 & 27 & 68 & 0 & 2 & 2 & 0 & 0 \\
\hline Leeds & 4 & 13 & 68 & 0 & 5 & 10 & 0 & 0 \\
\hline Leic & 3 & 24 & 55 & 0 & 8 & 10 & 0 & 0 \\
\hline Liv Ain & 3 & 12 & 82 & 0 & 0 & 2 & 0 & 0 \\
\hline Liv RI & 1 & 43 & 35 & 0 & 8 & 11 & 1 & 0 \\
\hline M Hope & 2 & 38 & 30 & 0 & 23 & 7 & 0 & 1 \\
\hline M RI & 19 & 19 & 33 & 0 & 6 & 19 & 4 & 0 \\
\hline Middlbr & 2 & 32 & 59 & 0 & 8 & 0 & 0 & 0 \\
\hline Newc & 4 & 80 & 0 & 0 & 3 & 14 & 0 & 0 \\
\hline Newry & 2 & 85 & 0 & 0 & 0 & 13 & 0 & 0 \\
\hline Norwch & 3 & 48 & 30 & 0 & 16 & 1 & 1 & 0 \\
\hline Nottm & 2 & 43 & 29 & 0 & 9 & 16 & 0 & 0 \\
\hline Oxford & 4 & 70 & 1 & 0 & 13 & 12 & 0 & 0 \\
\hline Plymth & 1 & 71 & 0 & 0 & 20 & 8 & 0 & 0 \\
\hline Ports & 0 & 29 & 53 & 0 & 18 & 0 & 0 & 0 \\
\hline Prestn & 5 & 22 & 60 & 0 & 5 & 8 & 0 & 0 \\
\hline Redng & 0 & 58 & 17 & 0 & 24 & 0 & 0 & 0 \\
\hline Sheff & 7 & 39 & 43 & 0 & 11 & 0 & 0 & 0 \\
\hline Shrew & 1 & 53 & 30 & 1 & 16 & 0 & 0 & 0 \\
\hline Stevng & 0 & 31 & 58 & 0 & 11 & 0 & 0 & 0 \\
\hline Sthend & 0 & 86 & 0 & 0 & 14 & 0 & 0 & 0 \\
\hline Stoke & 2 & 47 & 30 & 0 & 6 & 14 & 0 & 0 \\
\hline Sund & 1 & 65 & 21 & 0 & 8 & 6 & 0 & 0 \\
\hline Swanse & 4 & 67 & 13 & 0 & 16 & 0 & 0 & 0 \\
\hline Truro & 2 & 40 & 41 & 0 & 9 & 8 & 0 & 0 \\
\hline Tyrone & 1 & 90 & 0 & 0 & 1 & 8 & 0 & 0 \\
\hline Ulster & 1 & 91 & 1 & 0 & 0 & 6 & 0 & 0 \\
\hline Wirral & 2 & 32 & 45 & 0 & 7 & 14 & 0 & 0 \\
\hline Wolve & 0 & 22 & 60 & 0 & 18 & 0 & 0 & 0 \\
\hline Wrexm & 4 & 67 & 0 & 0 & 28 & 0 & 1 & 0 \\
\hline York & 1 & 54 & 32 & 0 & 13 & 0 & 0 & 0 \\
\hline England & 3 & 39 & 40 & 0 & 10 & 7 & 0 & $\mathbf{0}$ \\
\hline $\mathrm{N}$ Ireland & 2 & 85 & 0 & 0 & 2 & 11 & 0 & 0 \\
\hline Scotland ${ }^{*}$ & 3 & 81 & 0 & 0 & 6 & 9 & 1 & 0 \\
\hline Wales & 2 & 54 & 24 & 0 & 19 & 2 & 0 & 0 \\
\hline UK & 2 & 45 & 34 & 0 & 10 & 7 & 0 & 0 \\
\hline
\end{tabular}

Note:

Non-diabetic patients are calculated as all patients excluding diabetic patients and patients with a missing primary renal disease code Excluded centres with $\geqslant 40 \%$ primary renal diagnosis aetiology uncertain/glomerulonephritis (not biopsy proven) (Clwyd, Liverpool Aintree, Manchester Hope and Wirral)

* All haemodialysis patients in centres in Scotland are shown as receiving treatment in home or hospital as no information is available regarding numbers using satellite dialysis centres 
Table E.2.7. Number of non-diabetic patients by treatment modality

\begin{tabular}{lrrr}
\hline & HD & PD & Transplant \\
\hline England & 12,521 & 2,655 & 15,837 \\
N Ireland & 566 & 88 & 562 \\
Scotland & 1,450 & 282 & 1,797 \\
Wales & 765 & 206 & 917 \\
UK & $\mathbf{1 5 , 3 0 2}$ & $\mathbf{3 , 2 3 1}$ & $\mathbf{1 9 , 1 1 3}$ \\
\hline
\end{tabular}

\section{Note:}

Non-diabetic patients are calculated as all patients excluding diabetic patients and patients with a missing primary renal disease code Excluded centres with $\geqslant 40 \%$ primary renal diagnosis aetiology uncertain/glomerulonephritis (not biopsy proven) (Clwyd, Liverpool Aintree, Manchester Hope and Wirral)

Table E.2.8. Dialysis modalities for non-diabetic patients aged under 65

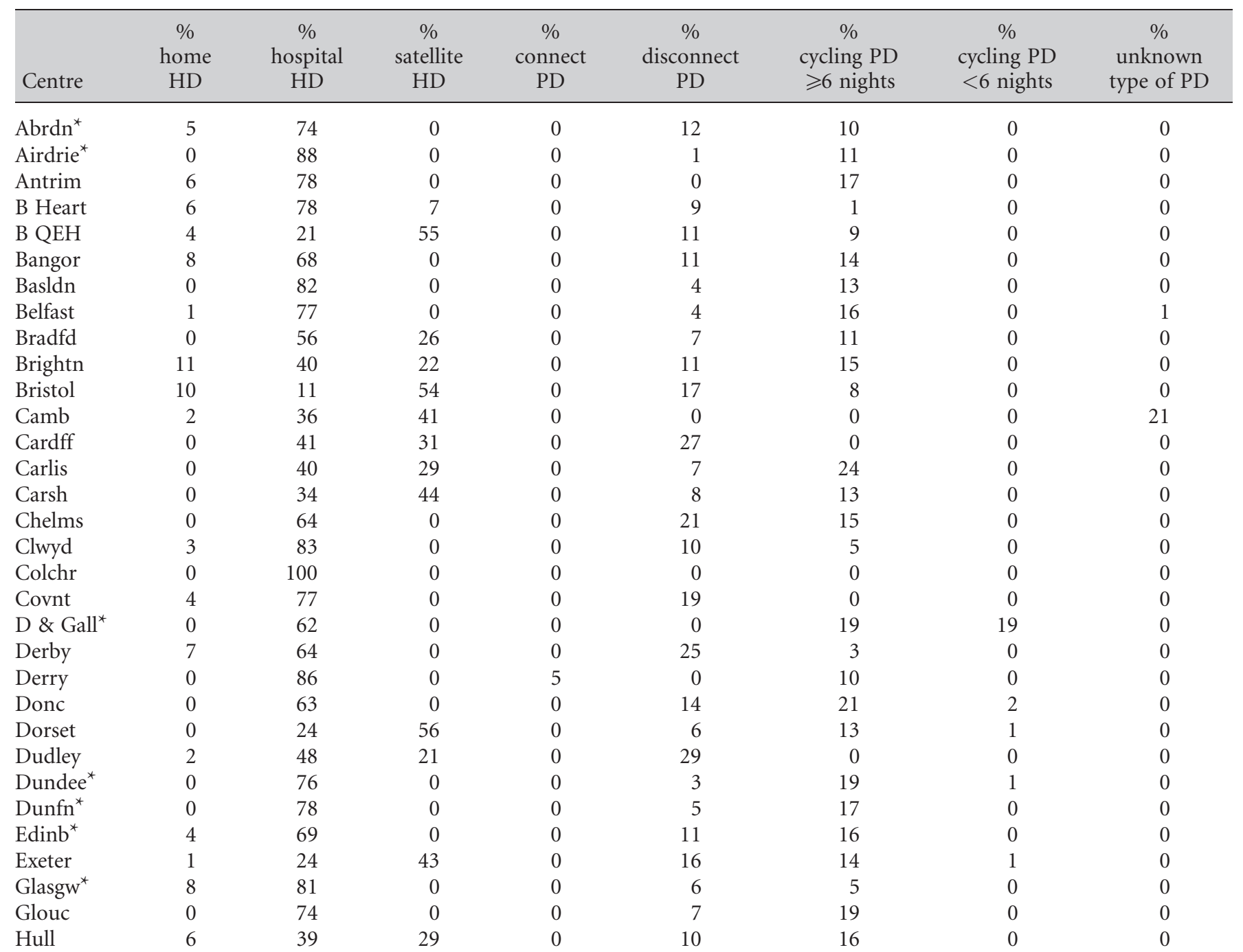


Table E.2.8. Continued

\begin{tabular}{|c|c|c|c|c|c|c|c|c|}
\hline Centre & $\begin{array}{c}\% \\
\text { home } \\
\text { HD }\end{array}$ & $\begin{array}{c}\% \\
\text { hospital } \\
\text { HD }\end{array}$ & $\begin{array}{c}\% \\
\text { satellite } \\
\text { HD }\end{array}$ & $\begin{array}{c}\% \\
\text { connect } \\
\text { PD }\end{array}$ & $\begin{array}{c}\% \\
\text { disconnect } \\
\text { PD }\end{array}$ & $\begin{array}{c}\% \\
\text { cycling PD } \\
\geqslant 6 \text { nights }\end{array}$ & $\begin{array}{c}\% \\
\text { cycling PD } \\
<6 \text { nights }\end{array}$ & $\begin{array}{c}\% \\
\text { unknown } \\
\text { type of PD }\end{array}$ \\
\hline Inverns ${ }^{*}$ & 7 & 64 & 0 & 0 & 7 & 23 & 0 & 0 \\
\hline Ipswi & 4 & 63 & 0 & 0 & 18 & 14 & 0 & 0 \\
\hline Kent & 2 & 17 & 55 & 0 & 26 & 0 & 0 & 0 \\
\hline Klmarnk $^{*}$ & 1 & 69 & 0 & 0 & 7 & 17 & 6 & 0 \\
\hline L Barts & 2 & 40 & 32 & 0 & 7 & 20 & 0 & 0 \\
\hline L Guys & 10 & 25 & 55 & 0 & 4 & 0 & 6 & 0 \\
\hline L Kings & 0 & 29 & 52 & 0 & 5 & 14 & 0 & 0 \\
\hline L Rfree & 5 & 37 & 44 & 0 & 4 & 9 & 0 & 0 \\
\hline L St.G & 5 & 63 & 11 & 2 & 5 & 14 & 0 & 0 \\
\hline L West & 2 & 28 & 66 & 0 & 2 & 3 & 0 & 0 \\
\hline Leeds & 7 & 14 & 59 & 0 & 7 & 12 & 0 & 0 \\
\hline Leic & 5 & 23 & 54 & 0 & 8 & 12 & 0 & 0 \\
\hline Liv Ain & 6 & 7 & 82 & 0 & 0 & 4 & 0 & 0 \\
\hline Liv RI & 2 & 38 & 39 & 0 & 6 & 14 & 0 & 0 \\
\hline M Hope & 3 & 34 & 28 & 0 & 25 & 9 & 0 & 1 \\
\hline M RI & 25 & 20 & 28 & 0 & 4 & 20 & 4 & 0 \\
\hline Middlbr & 3 & 34 & 53 & 0 & 10 & 1 & 0 & 0 \\
\hline Newc & 6 & 74 & 0 & 0 & 4 & 16 & 0 & 0 \\
\hline Newry & 2 & 76 & 0 & 0 & 0 & 22 & 0 & 0 \\
\hline Norwch & 5 & 47 & 23 & 0 & 24 & 0 & 1 & 0 \\
\hline Nottm & 3 & 43 & 22 & 0 & 9 & 23 & 0 & 0 \\
\hline Oxford & 6 & 65 & 1 & 0 & 10 & 18 & 0 & 0 \\
\hline Plymth & 2 & 62 & 0 & 0 & 21 & 16 & 0 & 0 \\
\hline Ports & 0 & 34 & 44 & 0 & 22 & 0 & 0 & 0 \\
\hline Prestn & 8 & 24 & 51 & 0 & 6 & 11 & 0 & 0 \\
\hline Redng & 1 & 50 & 16 & 0 & 33 & 0 & 0 & 0 \\
\hline Sheff & 11 & 35 & 44 & 0 & 11 & 0 & 0 & 0 \\
\hline Shrew & 1 & 43 & 34 & 1 & 21 & 0 & 0 & 0 \\
\hline Stevng & 0 & 32 & 53 & 0 & 14 & 0 & 0 & 0 \\
\hline Sthend & 0 & 76 & 0 & 0 & 24 & 0 & 0 & 0 \\
\hline Stoke & 4 & 42 & 33 & 0 & 5 & 17 & 0 & 0 \\
\hline Sund & 1 & 67 & 18 & 0 & 6 & 8 & 0 & 0 \\
\hline Swanse & 9 & 70 & 7 & 0 & 15 & 0 & 0 & 0 \\
\hline Truro & 3 & 38 & 31 & 0 & 16 & 13 & 0 & 0 \\
\hline Tyrone & 2 & 81 & 0 & 0 & 2 & 14 & 0 & 0 \\
\hline Ulster & 5 & 82 & 5 & 0 & 0 & 9 & 0 & 0 \\
\hline Wirral & 4 & 34 & 37 & 0 & 9 & 15 & 0 & 0 \\
\hline Wolve & 0 & 25 & 53 & 0 & 22 & 0 & 0 & 0 \\
\hline Wrexm & 7 & 73 & 0 & 0 & 20 & 0 & 0 & 0 \\
\hline York & 2 & 55 & 31 & 0 & 12 & 0 & 0 & 0 \\
\hline England & 4 & 38 & 37 & 0 & 11 & 9 & 0 & 0 \\
\hline N Ireland & 2 & 78 & 0 & 0 & 2 & 16 & 0 & 1 \\
\hline Scotland ${ }^{*}$ & 4 & 76 & 0 & 0 & 7 & 12 & 1 & 0 \\
\hline Wales & 4 & 57 & 17 & 0 & 20 & 1 & 0 & 0 \\
\hline UK & 4 & 44 & 31 & 0 & 11 & 9 & 0 & 0 \\
\hline
\end{tabular}

Note:

Non-diabetic patients are calculated as all patients excluding diabetic patients and patients with a missing primary renal disease code Excluded centres with $\geqslant 40 \%$ primary renal diagnosis aetiology uncertain/glomerulonephritis (not biopsy proven) (Clwyd, Liverpool Aintree, Manchester Hope and Wirral)

${ }^{\star}$ All haemodialysis patients in centres in Scotland are shown as receiving treatment in home or hospital as no information is available regarding numbers using satellite dialysis centres 
Table E.2.9. Number of non-diabetic patients aged under 65 by treatment modality

\begin{tabular}{lrrr}
\hline & HD & PD & Transplant \\
\hline England & 6,155 & 1,585 & 13,234 \\
N Ireland & 254 & 60 & 463 \\
Scotland & 743 & 184 & 1,532 \\
Wales & 338 & 98 & 773 \\
UK & $\mathbf{7 , 4 9 0}$ & $\mathbf{1 , 9 2 7}$ & $\mathbf{1 6 , 0 0 2}$ \\
\hline
\end{tabular}

Note:

Non-diabetic patients are calculated as all patients excluding diabetic patients and patients with a missing primary renal disease code Excluded centres with $\geqslant 40 \%$ primary renal diagnosis aetiology uncertain/glomerulonephritis (not biopsy proven) (Clwyd, Liverpool Aintree, Manchester Hope and Wirral)

Table E.2.10. Dialysis modalities for non-diabetic patients aged over 65

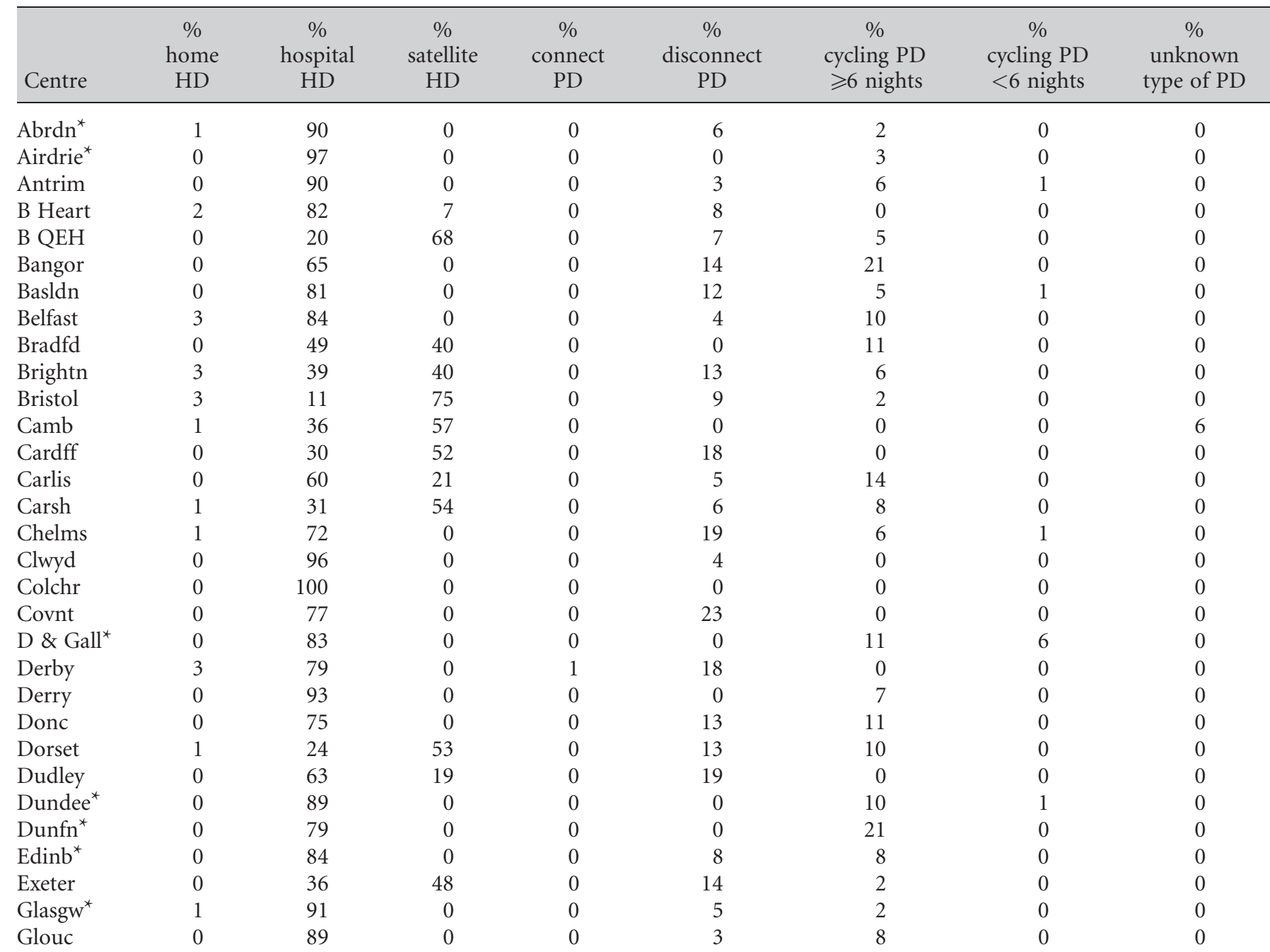


Table E.2.10. Dialysis modalities for non-diabetic patients aged over 65

\begin{tabular}{|c|c|c|c|c|c|c|c|c|}
\hline Centre & $\begin{array}{c}\% \\
\text { home } \\
\text { HD }\end{array}$ & $\begin{array}{c}\% \\
\text { hospital } \\
\text { HD }\end{array}$ & $\begin{array}{c}\% \\
\text { satellite } \\
\text { HD }\end{array}$ & $\begin{array}{c}\% \\
\text { connect } \\
\text { PD }\end{array}$ & $\begin{array}{c}\% \\
\text { disconnect } \\
\text { PD }\end{array}$ & $\begin{array}{c}\% \\
\text { cycling PD } \\
\geqslant 6 \text { nights }\end{array}$ & $\begin{array}{c}\% \\
\text { cycling PD } \\
<6 \text { nights }\end{array}$ & $\begin{array}{c}\% \\
\text { unknown } \\
\text { type of PD }\end{array}$ \\
\hline Hull & 2 & 39 & 44 & 0 & 6 & 9 & 0 & 0 \\
\hline Inverns* & 2 & 69 & 0 & 0 & 11 & 18 & 0 & 0 \\
\hline Ipswi & 0 & 63 & 0 & 0 & 25 & 11 & 0 & 2 \\
\hline Kent & 1 & 26 & 58 & 0 & 16 & 0 & 0 & 0 \\
\hline Klmarnk & 0 & 82 & 0 & 0 & 8 & 3 & 7 & 0 \\
\hline L Barts & 0 & 32 & 40 & 0 & 14 & 14 & 0 & 0 \\
\hline L Guys & 1 & 20 & 69 & 0 & 4 & 0 & 6 & 0 \\
\hline L Kings & 0 & 18 & 62 & 0 & 8 & 13 & 0 & 0 \\
\hline L Rfree & 1 & 35 & 55 & 0 & 3 & 5 & 0 & 0 \\
\hline L St.G & 0 & 59 & 20 & 4 & 7 & 9 & 0 & 0 \\
\hline L West & 0 & 26 & 70 & 0 & 2 & 2 & 0 & 0 \\
\hline Leeds & 0 & 11 & 79 & 0 & 3 & 7 & 0 & 0 \\
\hline Leic & 1 & 25 & 56 & 0 & 9 & 9 & 0 & 0 \\
\hline Liv Ain & 0 & 18 & 82 & 0 & 0 & 0 & 0 & 0 \\
\hline Liv RI & 0 & 51 & 30 & 0 & 12 & 5 & 3 & 0 \\
\hline M Hope & 0 & 43 & 32 & 0 & 21 & 3 & 0 & 1 \\
\hline M RI & 3 & 16 & 47 & 0 & 12 & 16 & 7 & 0 \\
\hline Middlbr & 1 & 30 & 64 & 0 & 6 & 0 & 0 & 0 \\
\hline Newc & 0 & 88 & 0 & 0 & 2 & 10 & 0 & 0 \\
\hline Newry & 2 & 94 & 0 & 0 & 0 & 4 & 0 & 0 \\
\hline Norwch & 1 & 50 & 35 & 0 & 11 & 2 & 1 & 0 \\
\hline Nottm & 2 & 43 & 38 & 0 & 8 & 8 & 0 & 0 \\
\hline Oxford & 2 & 75 & 0 & 0 & 16 & 6 & 0 & 0 \\
\hline Plymth & 0 & 77 & 0 & 0 & 20 & 3 & 0 & 0 \\
\hline Ports & 0 & 24 & 62 & 0 & 14 & 0 & 0 & 0 \\
\hline Prestn & 1 & 18 & 73 & 0 & 3 & 4 & 0 & 0 \\
\hline Redng & 0 & 66 & 18 & 0 & 16 & 0 & 0 & 0 \\
\hline Sheff & 3 & 43 & 43 & 0 & 11 & 0 & 0 & 0 \\
\hline Shrew & 0 & 62 & 27 & 0 & 11 & 0 & 0 & 0 \\
\hline Stevng & 0 & 30 & 61 & 0 & 8 & 0 & 0 & 0 \\
\hline Sthend & 0 & 94 & 0 & 0 & 6 & 0 & 0 & 0 \\
\hline Stoke & 0 & 53 & 27 & 0 & 8 & 12 & 0 & 0 \\
\hline Sund & 0 & 62 & 25 & 0 & 12 & 2 & 0 & 0 \\
\hline Swanse & 1 & 65 & 18 & 0 & 17 & 0 & 0 & 0 \\
\hline Truro & 1 & 42 & 46 & 0 & 6 & 6 & 0 & 0 \\
\hline Tyrone & 0 & 98 & 0 & 0 & 0 & 2 & 0 & 0 \\
\hline Ulster & 0 & 96 & 0 & 0 & 0 & 4 & 0 & 0 \\
\hline Wirral & 0 & 30 & 51 & 0 & 5 & 13 & 0 & 0 \\
\hline Wolve & 0 & 20 & 65 & 0 & 14 & 0 & 0 & 0 \\
\hline Wrexm & 2 & 63 & 0 & 0 & 33 & 0 & 2 & 0 \\
\hline York & 0 & 53 & 32 & 0 & 15 & 0 & 0 & 0 \\
\hline England & 1 & 41 & 44 & 0 & 9 & 5 & 0 & 0 \\
\hline N Ireland & 1 & 91 & 0 & 0 & 2 & 6 & 0 & 0 \\
\hline Scotland ${ }^{*}$ & 1 & 87 & 0 & 0 & 5 & 6 & 1 & 0 \\
\hline Wales & 0 & 51 & 29 & 0 & 18 & 2 & 0 & 0 \\
\hline UK & 1 & 47 & 38 & 0 & 9 & 5 & 0 & 0 \\
\hline
\end{tabular}

Note:

Non-diabetic patients are calculated as all patients excluding diabetic patients and patients with a missing primary renal disease code Excluded centres with $\geqslant 40 \%$ primary renal diagnosis aetiology uncertain/glomerulonephritis (not biopsy proven) (Clwyd, Liverpool Aintree, Manchester Hope and Wirral)

${ }^{*}$ All haemodialysis patients in centres in Scotland are shown as receiving treatment in home or hospital as no information is available regarding numbers using satellite dialysis centres 
Table E.2.11. Number of non-diabetic patients aged over 65 by treatment modality

\begin{tabular}{lrrr}
\hline & HD & PD & Transplant \\
\hline England & 6,366 & 1,070 & 2,603 \\
N Ireland & 312 & 28 & 99 \\
Scotland & 707 & 98 & 265 \\
Wales & 427 & 108 & 144 \\
UK & $\mathbf{7 , 8 1 2}$ & $\mathbf{1 , 3 0 4}$ & $\mathbf{3 , 1 1 1}$ \\
\hline
\end{tabular}

Note:

Non-diabetic patients are calculated as all patients excluding diabetic patients and patients with a missing primary renal disease code Excluded centres with $\geqslant 40 \%$ primary renal diagnosis aetiology uncertain/glomerulonephritis (not biopsy proven) (Clwyd, Liverpool Aintree, Manchester Hope and Wirral)

Table E.2.12. Dialysis modalities for diabetic patients

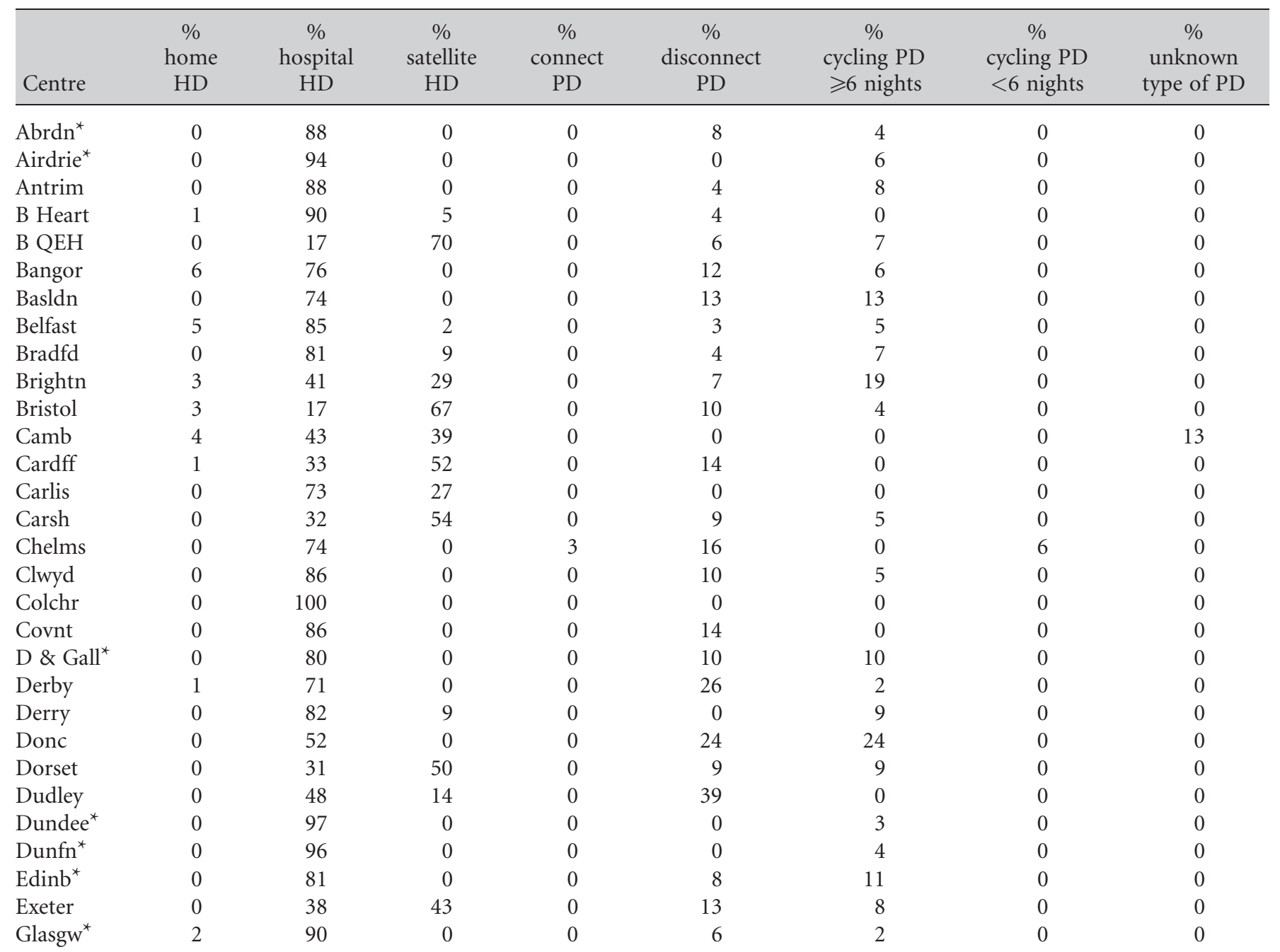


Table E.2.12. Dialysis modalities for diabetic patients

\begin{tabular}{|c|c|c|c|c|c|c|c|c|}
\hline Centre & $\begin{array}{c}\% \\
\text { home } \\
\text { HD }\end{array}$ & $\begin{array}{c}\% \\
\text { hospital } \\
\text { HD }\end{array}$ & $\begin{array}{c}\% \\
\text { satellite } \\
\text { HD }\end{array}$ & $\begin{array}{c}\% \\
\text { connect } \\
\text { PD }\end{array}$ & $\begin{array}{c}\% \\
\text { disconnect } \\
\text { PD }\end{array}$ & $\begin{array}{c}\% \\
\text { cycling PD } \\
\geqslant 6 \text { nights }\end{array}$ & $\begin{array}{c}\% \\
\text { cycling PD } \\
<6 \text { nights }\end{array}$ & $\begin{array}{c}\% \\
\text { unknown } \\
\text { type of PD }\end{array}$ \\
\hline Glouc & 0 & 83 & 0 & 0 & 4 & 13 & 0 & 0 \\
\hline Hull & 0 & 55 & 32 & 0 & 2 & 12 & 0 & 0 \\
\hline Inverns ${ }^{\star}$ & 0 & 91 & 0 & 0 & 4 & 4 & 0 & 0 \\
\hline Ipswi & 0 & 71 & 0 & 0 & 13 & 17 & 0 & 0 \\
\hline Kent & 1 & 24 & 57 & 0 & 18 & 0 & 0 & 0 \\
\hline Klmarnk $^{*}$ & 0 & 78 & 0 & 0 & 0 & 6 & 17 & 0 \\
\hline L Barts & 0 & 42 & 33 & 0 & 9 & 16 & 0 & 0 \\
\hline L Guys & 1 & 34 & 58 & 0 & 4 & 0 & 3 & 0 \\
\hline L Kings & 0 & 30 & 61 & 0 & 1 & 8 & 0 & 0 \\
\hline L Rfree & 0 & 46 & 46 & 0 & 2 & 5 & 0 & 0 \\
\hline L St.G & 0 & 60 & 23 & 0 & 3 & 13 & 0 & 0 \\
\hline L West & 0 & 31 & 67 & 0 & 1 & 1 & 0 & 0 \\
\hline Leeds & 1 & 15 & 71 & 0 & 5 & 9 & 0 & 0 \\
\hline Leic & 1 & 32 & 52 & 0 & 6 & 9 & 0 & 0 \\
\hline Liv Ain & 0 & 20 & 80 & 0 & 0 & 0 & 0 & 0 \\
\hline Liv RI & 0 & 39 & 35 & 0 & 11 & 15 & 0 & 0 \\
\hline M Hope & 0 & 38 & 62 & 0 & 0 & 0 & 0 & 0 \\
\hline M RI & 0 & 33 & 40 & 2 & 8 & 12 & 5 & 0 \\
\hline Middlbr & 0 & 33 & 61 & 0 & 6 & 0 & 0 & 0 \\
\hline Newc & 0 & 85 & 0 & 0 & 0 & 15 & 0 & 0 \\
\hline Newry & 0 & 100 & 0 & 0 & 0 & 0 & 0 & 0 \\
\hline Norwch & 0 & 49 & 37 & 0 & 12 & 0 & 2 & 0 \\
\hline Nottm & 0 & 58 & 23 & 0 & 10 & 9 & 0 & 0 \\
\hline Oxford & 0 & 73 & 1 & 0 & 13 & 12 & 0 & 0 \\
\hline Plymth & 0 & 68 & 0 & 0 & 12 & 20 & 0 & 0 \\
\hline Ports & 0 & 50 & 36 & 0 & 14 & 0 & 0 & 0 \\
\hline Prestn & 4 & 24 & 64 & 0 & 2 & 7 & 0 & 0 \\
\hline Redng & 0 & 56 & 21 & 0 & 23 & 0 & 0 & 0 \\
\hline Sheff & 2 & 39 & 44 & 0 & 15 & 0 & 0 & 0 \\
\hline Shrew & 0 & 49 & 33 & 0 & 18 & 0 & 0 & 0 \\
\hline Stevng & 0 & 30 & 64 & 0 & 6 & 0 & 0 & 0 \\
\hline Sthend & 0 & 100 & 0 & 0 & 0 & 0 & 0 & 0 \\
\hline Stoke & 0 & 49 & 22 & 0 & 10 & 19 & 0 & 0 \\
\hline Sund & 0 & 78 & 15 & 0 & 5 & 3 & 0 & 0 \\
\hline Swanse & 3 & 67 & 12 & 0 & 18 & 0 & 0 & 0 \\
\hline Truro & 0 & 33 & 52 & 0 & 11 & 4 & 0 & 0 \\
\hline Tyrone & 0 & 91 & 0 & 0 & 0 & 9 & 0 & 0 \\
\hline Ulster & 0 & 95 & 0 & 0 & 0 & 5 & 0 & 0 \\
\hline Wirral & 0 & 20 & 72 & 0 & 0 & 8 & 0 & 0 \\
\hline Wolve & 0 & 35 & 51 & 0 & 13 & 1 & 0 & 0 \\
\hline Wrexm & 0 & 87 & 0 & 0 & 13 & 0 & 0 & 0 \\
\hline York & 0 & 40 & 40 & 0 & 20 & 0 & 0 & 0 \\
\hline England & 0 & 44 & 41 & 0 & 8 & 6 & 0 & 0 \\
\hline N Ireland & 2 & 89 & 1 & 0 & 2 & 6 & 0 & 0 \\
\hline Scotland ${ }^{\star}$ & 1 & 89 & 0 & 0 & 5 & 5 & 1 & 0 \\
\hline Wales & 2 & 53 & 31 & 0 & 14 & 1 & 0 & 0 \\
\hline UK & 1 & 49 & 36 & 0 & 8 & 6 & 0 & 0 \\
\hline
\end{tabular}

Note: Diabetic patients are patients with a primary renal disease code of diabetes

${ }^{*}$ All haemodialysis patients in centres in Scotland are shown as receiving treatment in home or hospital as no information is available regarding numbers using satellite dialysis centres 
Table E.2.13. Number of diabetic patients by treatment modality

\begin{tabular}{lrrr}
\hline & HD & PD & Transplant \\
\hline England & 3,314 & 575 & 1,565 \\
N Ireland & 153 & 14 & 47 \\
Scotland & 319 & 37 & 173 \\
Wales & 224 & 40 & 113 \\
UK & $\mathbf{4 , 0 1 0}$ & $\mathbf{6 6 6}$ & $\mathbf{1 , 8 9 8}$ \\
\hline
\end{tabular}

Note: Diabetic patients are patients with a primary renal disease code of diabetes

Table E.2.14. Diabetics

\begin{tabular}{|c|c|c|c|c|c|}
\hline Centre & $\mathrm{M}: \mathrm{F}$ ratio & $\begin{array}{c}\text { Median age on } \\
31 / 12 / 2008\end{array}$ & $\begin{array}{l}\text { Median age at } \\
\text { start of treatment }\end{array}$ & $\begin{array}{l}\text { Median time on } \\
\text { RRT in days }\end{array}$ & $\begin{array}{l}\text { Median time on } \\
\text { RRT in years }\end{array}$ \\
\hline Abrdn & 1.3 & 58 & 54 & 1,374 & 3.8 \\
\hline Antrim & 1.1 & 65 & 60 & 1,226 & 3.4 \\
\hline B Heart & 1.8 & 65 & 61 & 959 & 2.6 \\
\hline $\mathrm{B} \mathrm{QEH}$ & 1.5 & 65 & 60 & 968 & 2.7 \\
\hline Belfast & 1.8 & 58 & 55 & 1,289 & 3.5 \\
\hline Bradfd & 1.8 & 62 & 57 & 897 & 2.5 \\
\hline Brightn & 1.8 & 62 & 59 & 971 & 2.7 \\
\hline Bristol & 1.3 & 61 & 56 & 992 & 2.7 \\
\hline Camb & 2.4 & 46 & 40 & 1,723 & 4.7 \\
\hline Cardff & 1.7 & 61 & 57 & 962 & 2.6 \\
\hline Colchr & 1.3 & 65 & 60 & 1,525 & 4.2 \\
\hline Covnt & 1.6 & 64 & 60 & 886 & 2.4 \\
\hline D \& Gall & 2.2 & 59 & 52 & 1,127 & 3.1 \\
\hline Derby & 1.5 & 63 & 61 & 899 & 2.5 \\
\hline Derry & 0.6 & 55 & 48 & 1,512 & 4.1 \\
\hline Donc & 3.0 & 55 & 50 & 987 & 2.7 \\
\hline Dorset & 2.3 & 56 & 52 & 1,036 & 2.8 \\
\hline Dudley & 3.3 & 64 & 59 & 874 & 2.4 \\
\hline Dundee & 1.3 & 59 & 54 & 1,254 & 3.4 \\
\hline Dunfn & 0.6 & 54 & 49 & 763 & 2.1 \\
\hline Edinb & 1.6 & 58 & 55 & 950 & 2.6 \\
\hline Exeter & 1.3 & 55 & 51 & 1,314 & 3.6 \\
\hline L Barts & 1.9 & 62 & 58 & 910 & 2.5 \\
\hline L Guys & 1.4 & 56 & 51 & 1,156 & 3.2 \\
\hline L Kings & 1.3 & 64 & 61 & 973 & 2.7 \\
\hline L Rfree & 1.3 & 65 & 60 & 1,036 & 2.8 \\
\hline L St.G & 1.4 & 68 & 62 & 1,318 & 3.6 \\
\hline
\end{tabular}


Table E.2.14. Continued

\begin{tabular}{|c|c|c|c|c|c|}
\hline Centre & $\mathrm{M}: \mathrm{F}$ ratio & $\begin{array}{c}\text { Median age on } \\
31 / 12 / 2008\end{array}$ & $\begin{array}{l}\text { Median age at } \\
\text { start of treatment }\end{array}$ & $\begin{array}{l}\text { Median time on } \\
\text { RRT in days }\end{array}$ & $\begin{array}{l}\text { Median time on } \\
\text { RRT in years }\end{array}$ \\
\hline L West & 1.6 & 61 & 57 & 1,288 & 3.5 \\
\hline Leeds & 1.1 & 60 & 54 & 1,705 & 4.7 \\
\hline Leic & 1.7 & 60 & 55 & 997 & 2.7 \\
\hline Liv Ain & 0.7 & 68 & 62 & 1,978 & 5.4 \\
\hline Liv RI & 1.6 & 56 & 51 & 1,646 & 4.5 \\
\hline M Hope & 1.2 & 61 & 56 & 2,259 & 6.2 \\
\hline M RI & 1.4 & 56 & 49 & 1,593 & 4.4 \\
\hline Middlbr & 1.1 & 52 & 47 & 1,125 & 3.1 \\
\hline Newc & 1.4 & 53 & 48 & 1,267 & 3.5 \\
\hline Newry & 1.9 & 65 & 60 & 1,247 & 3.4 \\
\hline Norwch & 1.3 & 64 & 60 & 827 & 2.3 \\
\hline Nottm & 1.1 & 61 & 56 & 990 & 2.7 \\
\hline Oxford & 1.7 & 55 & 49 & 1,370 & 3.8 \\
\hline Plymth & 1.6 & 56 & 53 & 1,057 & 2.9 \\
\hline Ports & 1.8 & 56 & 53 & 820 & 2.2 \\
\hline Prestn & 2.0 & 63 & 59 & 1,040 & 2.8 \\
\hline Redng & 1.9 & 62 & 59 & 874 & 2.4 \\
\hline Sheff & 2.0 & 61 & 55 & 1,044 & 2.9 \\
\hline Shrew & 1.8 & 63 & 60 & 786 & 2.2 \\
\hline Stevng & 1.9 & 62 & 60 & 824 & 2.3 \\
\hline Sthend & 1.7 & 64 & 57 & 1,003 & 2.7 \\
\hline Stoke & 1.1 & 61 & 57 & 1,189 & 3.3 \\
\hline Sund & 1.8 & 57 & 55 & 910 & 2.5 \\
\hline Swanse & 1.9 & 62 & 58 & 775 & 2.1 \\
\hline Truro & 2.0 & 61 & 62 & 826 & 2.3 \\
\hline Tyrone & 0.8 & 64 & 60 & 1,394 & 3.8 \\
\hline Ulster & 1.4 & 67 & 65 & 852 & 2.3 \\
\hline Wirral & 1.3 & 55 & 53 & 897 & 2.5 \\
\hline Wolve & 2.0 & 59 & 56 & 944 & 2.6 \\
\hline Wrexm & 3.7 & 53 & 42 & 1,803 & 4.9 \\
\hline York & 1.6 & 55 & 53 & 1,002 & 2.7 \\
\hline England & 1.6 & 60 & 56 & 1,065 & 2.9 \\
\hline N Ireland & 1.4 & 62 & 58 & 1,226 & 3.4 \\
\hline Scotland & 1.4 & 56 & 52 & 1,127 & 3.1 \\
\hline Wales & 1.9 & 61 & 57 & 912 & 2.5 \\
\hline UK & 1.6 & 60 & 56 & 1,059 & 2.9 \\
\hline
\end{tabular}

Note:

Diabetic patients are patients with a primary renal disease code of diabetes

Patients with an initial treatment modality of transferred in or transferred out were excluded from the calculation of median age at start of treatment and median time on RRT, since their treatment start date is not accurately known

Table E.2.15. Transplant gender ratios

\begin{tabular}{lccccc}
\hline & \% males & \% females & No. males & No. females & M:F ratio \\
\hline England & 60.5 & 39.5 & 11,238 & 7,325 & 1.5 \\
N Ireland & 63.6 & 36.4 & 388 & 222 & 1.7 \\
Scotland & 59.2 & 40.8 & 1,171 & 808 & 1.4 \\
Wales & 63.2 & 36.8 & 725 & 423 & 1.7 \\
UK & $\mathbf{6 0 . 6}$ & $\mathbf{3 9 . 4}$ & $\mathbf{1 3 , 5 2 2}$ & $\mathbf{8 , 7 7 8}$ & $\mathbf{1 . 5}$ \\
\hline
\end{tabular}

